\title{
Patient Centered Design: Challenges and Lessons Learned from Working with Health Professionals and Schizophrenic Patients in e-Therapy Contexts
}

\author{
Catarina I. Reis ${ }^{1}$, Carla S. Freire ${ }^{2}$, Joaquin Fernández ${ }^{3}$, \\ and Josep M. Monguet ${ }^{3}$ \\ ${ }^{1}$ Department of Computer Science, School of Technology and Management, \\ Polytechnic Institute of Leiria, Portugal \\ catarina.reis@ipleiria.pt \\ ${ }^{2}$ Department of Mathematics and Natural Sciences, School of Education, \\ Polytechnic Institute of Leiria, Portugal \\ carla.freire@ipleiria.pt \\ ${ }^{3}$ Department of Graphic Expression \\ Polytechnical University of Catalonia, Spain \\ \{ifernandez,jm.monguet\}@upc.edu
}

\begin{abstract}
Patient Centered Design (PCD) is a particular type of User Centered Design (UCD) where the end-user is a patient that will use an Information and Communications Technology (ICT) solution for healthcare. It focuses on needs, wants and skills of the product's primary user and implies involving end-users in the decision-making and development process of the solution. e-Therapy aims to provide support to therapy sessions through ICT solutions. It has grown in the last years and in the mental health arena is being used for specific therapeutic contexts. It is an especially difficult environment due to specificities of the patients' conditions and where the physical access to patients is restricted and, sometimes, not even possible. Thus, a PCD approach can be accomplished through the health professionals involved, applying some of the most wellknown methods of UCD: interviews, questionnaires, focus groups and participatory design. eSchi is an e-Therapy tool that complements traditional practices for the cognitive rehabilitation and training of schizophrenic patients. It was successfully developed using a PCD approach.
\end{abstract}

Keywords: Patient Centered Design (PCD), User Centered Design (UCD), Information and Communications Technology (ICT), e-Therapy, Mental Health, Interviews, Questionnaires, Focus Group, Participatory Design, eSchi, Schizophrenia.

\section{Introduction}

Mental disorders such as schizophrenia are amongst the 20 leading causes of disability worldwide [55]. Schizophrenia is one of the disorders identified as a priority. Currently, it affects about seven per thousand of the world adult population, mostly in the age group 15-35 years, summing up to almost 24 million people 
worldwide. Although the incidence rate is rather low, three per 10.000 inhabitants, the prevalence is high due to chronicity [53] and [54].

Schizophrenia is a severe mental disorder usually detected in the early adulthood and characterized by disruptions in thinking. People find difficult "to tell the difference between real and unreal experiences, to think logically, to have normal emotional responses, and to behave normally in social situations" [33]. Someone that endures this condition sees his/her language, perception, and sense of self, affected. Seldom, the condition includes psychotic experiences, such as hearing voices or delusions, and can impair functioning through the loss of an acquired capability, disabling the person to earn a living or continue with its studies.

There is no known cure for schizophrenia, but it is treatable and allows some of those who suffer from it to have an adequate and productive life, enabling them to integrate fully into society. Severe cognitive deficits such as memory loss are a reality for schizophrenia patients, regardless of the schizophrenia' subtype considered: paranoid; hebephrenic or disorganized; catatonic; undifferentiated or residual [16].

Current national policies are especially dedicated to prevent and improve mental health assistance, considering mental disorders as a public health case. One of these policies includes the digital provision of mental health (e-Mental Health) information and services through Mental Health Information Systems (MHIS) [52] and [53].

e-Therapy is a subsystem of an MHIS that enables the electronic provision of an already existing and specific health service: therapy. It "is a new modality of helping people resolve life and relationship issues. It uses the power and convenience of the Internet to allow simultaneous (synchronous) and time-delayed (asynchronous) communication between an individual and a professional" for Castelnuovo et al. [7]. In the e-Mental Health context there are many actors involved and contributing to the global welfare such as patients, families, caregivers and health professionals such as psychiatrists, therapists and psychologists [30]. e-Therapy may have an important role contributing not only to the patient's treatment but also in the education process of all the actors involved. According to the Institute of Medicine's report, Crossing the Quality Chasm, developing “patient-specific clinical information”" computer systems is critical to improve the care of individuals with a chronic illness such as a severe mental illness (SMI) [10]. Computer-guided therapy is an innovative strategy that can play an important role in the future of psychological treatment [34], [39]. When this option is available to patients, they are free to accept/reject it with all the benefits of traditional Cognitive Behavior Therapy (CBT) available in a computer as a complement.

An essential feature of the systems mentioned above is the feedback provided to the users. Experts in the field often debate what produces effective feedback; but they all agree that continuous monitoring of the patients' treatment response and provision of the information to the treating clinician are vital.

\section{Patient Centered Design}

User Centered Design (UCD) is a technique based on three principles: focus on users and tasks, measure usability empirically and develop usability iteratively [13] and 
[51]. Patient Centered Design (PCD) is a particular type of UCD where the end-user is a patient that will use a healthcare solution that should meet his/her expectations [14]. Several standards relate to these design approaches and they provide practical recommendations to apply in the development of interactive systems, the software engineering approach to take when considering the usability and quality of software products and its corresponding evaluation.

\subsection{User Centered Design}

In the software development industry, the major aim is to obtain a high quality product that satisfies its end-users. To achieve this goal it is essential to first define what "high quality" means and then, make the product according to it. Usability is a high-level quality objective and serves as the base principle for User-Centered Design (UCD) as we shall see.

The industry has already proven, like IBM and Google for instance, that the best reason for using UCD is that “if the user can't use it, it doesn't work" (attributed to Susan Dray by [2]). "People ignore design that ignores people" is a saying by Frank Chimero [9] that empowers the key differentiator of developing software that is the “easy-of-use” [5], [12], [20], [32], [35], [42].

It might seem obvious that users should be involved directly in projects, but when real-life projects are concerned, several difficulties arise [8]. For instance, many organizations do not seem to realize the cost-benefit of involving the users, right from the start of the software development process. According to some authors, UCD has cost benefits, while according to others [6], [27] the usability evaluation methods are not cost effective.

Several ISO standards are defined and currently in use in the industry. IBM, Apple and Google are some of the companies, to name a few, that use them and are widely known and successful. They seek to provide effective, efficient and satisfying products for use in a specific context. This requires the design of an appropriate interaction and interface, achieved through a user-centered process that is only viable with a consistent organizational capability. IBM is rearranging their development methodologies to lodge the usability experience centered in the final users. Now, the User Experience and Design (UXD) professionals have to adapt their tasks, activities and deliverables to center their work in the users' feedback and iterative development. As far as Google is concerned, in 2007 its User eXperience (UX) experts' team published a set of Googley Design Principles that defines smart design approaches. The ten published principles flow from well-known facts acquired by Google's experience and the UX team' stated mission: “design products that satisfy and delight our users". Thus, a product that accomplishes a harmonious balance between being useful, fast, simple, engaging, innovative, universal, profitable, beautiful, trustworthy and personable, is considered a Googley product [18].

The most referred standards related to usability are the ISO 9241 series [21]. These series are concerned with describing usability and the ergonomics in a specific context of use. They include the human-system interaction of the systems and the guidelines specifically for the design of accessible software including the elderly and persons with disabilities. 
Usability is an important aspect of software products. Probably the most used definition on usability is by Nielsen and dates from 1993: "usability is about learnability, efficiency, memorability, errors, and satisfaction" [35]. The current standard definition of usability introduced in 1998 is as follows: "the extent to which a product can be used by specified users to achieve specified goals with effectiveness, efficiency and satisfaction in a specified context of use" [22].

While ISO 9241 defines usability, another standard, the ISO 13407 refers to specificities of designing usability, the process of achieving usable systems [25]. The product/system to be developed has humans/users in the center of the operations. Established since 1999, this standard aims to provide guidance into how to develop interactive computer systems centered on its future users. The idea is to accomplish a usable system.

There is relevant work done that discusses the usefulness and characteristics of ISO 13407 [15], [24], [31]. Reduced training and support costs, user satisfaction and productivity guarantees are some of the benefits depicted in the standard's rationale. All the previous referred work agrees on the fact that the standard is for those who manage design processes and does not provide detailed information of methods and techniques [4]. Nevertheless, the standard identifies the following four general principles that characterize the user-centered design:

- the active involvement of users and a clear understanding of user and task requirements,

- an appropriate allocation of functions between users and technology,

- iteration of design solutions,

- and multi-disciplinary design.

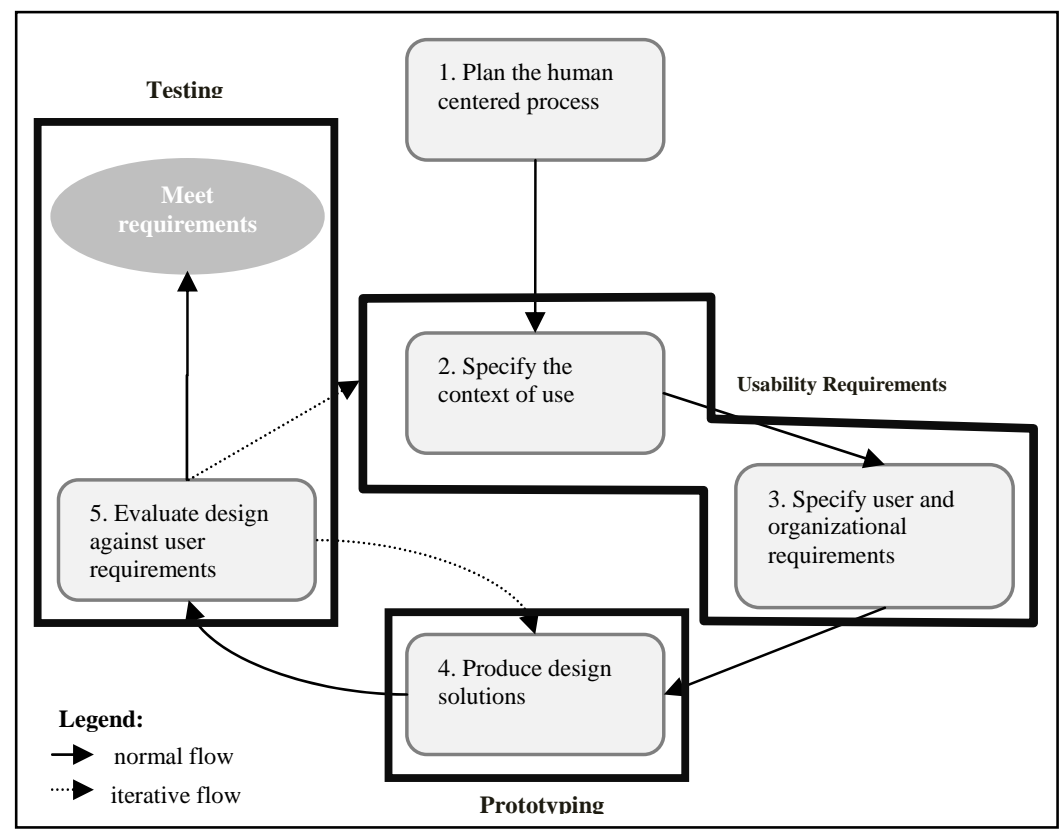

Fig. 1. User Centered Design Process (adapted from [4]) 
The UPA - Usability Professional's Association - defines a typical UCD methodology named "Designing the User Experience" based on the principle: "Providing a great user experience is an ongoing process" [49]. In this methodology, UCD activities appear arranged into the four typical software development phases: analysis, design, implementation and deployment (Fig. 1.).

After identifying the need for human-centered design, the designer team should plan which methods to use during the distinct phases of the approach (step one). Teamwork and communication are extremely important. Knowing the environment where the product will be used is essential for a good outcome (step two). In order to determine the tasks that users should accomplish when using the product (step three), the team should define users and personnel involved in the system and this specification can be achieved using direct user observation or contextual inquiry [13], [29], participatory design [11], [19], [23], interviews, questionnaires [1], focus groups or brainstorming.

Several usability inspection methods, including heuristic evaluation, user satisfaction methods, and the performance measurement method evaluate the proposed design (step 5). Nielsen's Heuristic Evaluation [35] is a method for usability testing that enables the identification of problems supported by factor principles. It is a method extremely easy to learn and inexpensive to use.

The whole idea in testing is to assess the degree of achievement of requirements. In order to answer a simple question: “does this design support the user's tasks?" it is required to diagnose eventual usability problems and evaluate the achievement of the objectives. Testers often do not have the knowledge, instruments and/or time available to test for usability [40].

On the other hand, narratives and explanations of study participants through the think-aloud method or post-study open-ended interviews are some of the techniques mostly used for these systems' evaluation. There is still much work to be done, moments of reflection and observations of changes in work practices or home routines are extremely hard to capture [47].

\subsection{Patient Centered Design for Mental Health Contexts}

Patient Centered Design (PCD) is the designation of a methodology that implies involving patients in the decision-making and development process of an ICT solution. When using such a methodology, patient empowerment occurs and they start to have an active role that allows them to make choices and provide input regarding their treatments [14].

This approach to design is tightly linked to the current development of patientcentered healthcare delivery processes [13]. However, introducing ICT in clinical settings should be done cautiously, namely to support clinicians tasks and augment the services' delivery to patients [40], [43]. The main idea is to replace pen and paper for computers and digital media, having most of these services provided to the citizens through the Internet [37]. In fact, this approach is a fundamental piece to provide Healthcare ICT solutions and implies partnerships between practitioners, patients and families/caretakers [14]. Some identify the partnerships as clinical microsystems where everyone is responsible for the actual care conditions, patients 
included. These liaisons and their nature will have a direct impact on the social context for the product implementation and its complete acceptance, later. The product is an artifact that results from the social exchanges in the group and is close to the users [46]. This multiplicity of users usually implies multiple user interfaces and plenty to describe regarding approaches to the design and development of such systems [44].

In summary, PCD is a particular type of User Centered Design (UCD) where the end-user is a patient that will use a healthcare solution that should meet his/her expectations. UCD focus on needs, wants, skills and preferences of the product's primary user. Thus, as a specific type of UCD, PCD starts by listening to the patient's needs and requirements besides considering the social and technical context for the implementation of the product. Most failures come from the absence of feedback from end-users and success is achieved when product designers attend to the needs and wants of users, engaging them early in the design and test process. Human factors engineering knowledge should not be ignored; neither assumed that the already acquired knowledge is enough. To develop a better fitting organizational system and discover ways to achieve the proposed goal, user involvement is as important as it is necessary. Such a system should meet several goals as well as serve distinct types of users, while ensuring that their work routines are available [37], [46].

PCD should be the central point of a system implementation engineering process, based on existing guidelines for software development. Several approaches gather the vital knowledge needed to prevent rejection of the system, and reduce the information asymmetry usually existent in the implementation process. Few have used a PCD approach in the development of ICT healthcare solutions [3], [46].

Benefits of PCD include the increase of the communication between the healthcare figures. Involvement of clinicians and other staff in the system development and in human computer interface design is crucial for success. There should be an interest in linking organization goals with the user goals and the features delivered by the system.

Examples of undesirable outcomes include missing deadlines, poor user adoption and not achieving the expected benefits. Understanding the risks and prioritizing them enables the development team to conduct proper risk management [38], [45]. Amongst the findings of the referred studies, the risks considered most important include the importance of a project champion, the lack of commitment from upper management, the perceived usefulness associated with use of an information system, the project ambiguity and the result of the quality of alignment between the system characteristics and the organization of clinical work.

When it comes to the mental health arena, there are already some services available in the field, but there is no evidence of a sound and systematic method to develop ICT for schizophrenia or psychosis patients [13], [26], [50].

\section{3 eSchi - an e-Therapy solution}

eSchi is a web-enabled multimedia system intended for use in therapy settings. The system provides a commonplace for patients, health professionals and caregivers to relate and communicate with each other. eSchi, amongst other functionalities, allows 
the management of patients and therapy sessions, as well as carrying out sessions and visualizing the results obtained in those sessions. While patients can conduct cognitive-related activities, where the system plays a more entertaining role, health professionals can monitor and visualize the patients' performance in e-Therapy settings.

The eSchi system contains a set of multimedia tools, available online, that enable schizophrenic patients' cognitive enhancement [17]. The tools help patients in their cognitive rehabilitation and therapists in their work. The main idea was to develop an e-Therapy tool that can be seen as a learning tool to teach and train schizophrenic patients to acquire basic skills that were once lost because of the cognitive impairments suffered.

eSchi is designed as a modular system with two separate modules: the patient module and the therapist module. While the patient module relates to aspects concerning the learning and training of cognitive functions of the patients, the therapist module is more dedicated to the management issues regarding patients and sessions.

Patients will first have to train their motion skills with simple games for the usage of the mouse: moving objects around, clicking on specific places and dragging and dropping objects. Accurate data regarding the patient performance, during the training is recorded. Hence, it is possible to know the patient behavior during a specific activity. There are also basic cognition activities related to recognition and association of objects. In a recognition activity, a stimulus object is shown to the patient for some seconds and after that time, the stimulus disappears. The patient is presented with a set of distinct stimuli, and he/she must identify the stimulus he/she has seen before. In an association activity, a stimulus object is shown associated with other stimulus (for instance, milk associated with the color yellow). When these stimuli disappear, the patient will be presented with unrelated stimuli and the patient must identify the previous relationship between stimuli.

Therapists are able to manage information regarding patients' data, see patients' performance and configure sessions and activities for the patients. The therapist may choose an easier or more complex activity, with more or less time dedicated per activity, according to the type of patient. After conducting the session with the patient, the therapist is able to see the patients' performance during a specific period of time and program future sessions according to the observed results.

The eSchi system was analyzed, designed and implemented using a PCD approach that we will present in the topic of Challenges and Lessons Learned.

\subsection{Fieldwork}

We had the privilege to deploy eSchi and conduct our study and test it, under real clinical settings. This is something incredible difficult to achieve in the mental health context [48]. The fieldwork was planned recurring to the well-known case study methodology proposed by Yin [56], [57] and later expanded by Kitchenham [28]. Two case studies, similar to a sister-study, were defined and shared the case study design. Thus, several aspects of the study were kept with minor changes. 
Case Study at Hospital Sant Joan de Déu. The medical team of the Hospital Sant Joan de Déu was a part of the development team of eSchi. The initial set of multimedia activities was defined with the help of that team and the actual implementation of the system in the setting was thought has being transparent. The base language support for the eSchi system was Spanish. Sant Joan de Déu is a religious order that started back in the XVI century and that through the existence of many workers, collaborators, volunteers and benefactors, assists and helps the ill and the disadvantaged. The Hospital Sant Joan de Déu is located close to the city of Barcelona in Sant Boi de Llobregat and Esplugues de Llobregat.

Case Study at Hospital Magalhães de Lemos. The Hospital Magalhães de Lemos (HML) is considered as a psychiatric-specialized hospital and is the only one that serves the north of Portugal. The hospital directs its available services to anyone that carries a severe and chronic mental illness; and with difficulties in the psychosocial functioning and community integration. It ensures the provision of rehabilitation services to patients and social reintegration to those that have no familiar back support and have been living in the facilities for long periods. The service has a multidisciplinary team and enables the acquisition of psychosocial skills by the patient allowing him to feel more confident and happy with his/her social role.

Design of both case studies. The first step was to conceive an implementation protocol plan that defined the steps to follow in the usage of the eSchi system. The protocol contained a brief explanation of the procedures and a plan with the needed resources: human and temporal. It proposed a three-week period intervention subdivided into three distinct and sequential steps, relative to the usage of the system. First, in the pre-usage phase, the users answered a questionnaire that will help identify their expectations regarding the usage of the system and obtain some demographic data. This occurs in the same day that the next phase (usage) starts. This second phase corresponds to a three-week period with a frequency of one session per day. During this phase, a psychiatrist conducts its patients' sessions using the eSchi system as a complement in the therapy and developing cognitive activities. After each session is completed, all the users answered to a simple questionnaire regarding their opinion and satisfaction with the usage of the system throughout the session. At last, in the after-usage phase, both patients and psychiatrists, involved in the study, were invited to answer to two questionnaires that helped in the identification of their opinion and general satisfaction regarding their usage of the eSchi system. This last step occurred in two distinct moments in time: in the same day of the last session and after a twoweek interval, without using the tool.

\section{$4 \quad$ Challenges and Lessons Learned}

The eSchi system was implemented, deployed and data was collected from two distinct case studies. Despite the efforts to minimize existing confounding factors that might influence the results collected, the perfect environment could not be achieved. The idea was to provide two similar sites in which the system would be implemented but reality is actually quite different. It is almost impossible to ensure the same type 
of conditions from site to site. Each site has its own unique conditions that include aspects such as an internment ward or an ICT laboratory, schizophrenia typologies present... The number of patients that engaged in the study was considerable different, from site to site, and even the diagnosis, the typologies of schizophrenia and schizoaffective conditions were distinct.

"There are no illnesses, only patients" is a popular saying in the mental health area. Each patient has its own individual and unique condition and cannot, and should not, be grouped into a cluster either in diagnose or treatment. This is the belief of all the health professionals in the mental health field that we had the opportunity to meet. The patient individuality should be respected and this, sometimes, implies a particular treatment protocol. These concerns prove to be highly considerate for the patients and improve the environment lived in the facilities. Nevertheless, this is also one if not the major causes for the harsh situations found when trying to conduct valid research studies, such as this one. Research usually provides new and additional insights on some patterns and characteristics groups, helping in the science discovery process.

Despite the fact that schizophrenia is a chronic illness that, at present, has no scientific evidence on cognition improvements, the indicators collected by the system attempt to provide some insight into the patient's behavior within a session and throughout a sequence of episodes in time.

The results seem to show that patients seem to develop their ability through training. Repetition of the same activities though a certain period seems to improve the outcomes. The actual time spent on an activity while trying to accomplish the specified goals helps to the validation of the other indicators. After some sessions, patients seem to stabilize the amount of time they spend on a session and actually, the effective time seems to decrease over time.

Another aspect worth mentioning is the fact that it appears to be a direct relationship between the user ASQ score and the average performance demonstrated in the system's usage. Thus, since there seems to be some redundancy on the information, the ASQ answering could possibly be replaced by the data collection analysis enabled by eSchi.

There was some additional feedback worth mentioning. Some free-text comments, mainly made by the therapists, requested new and improved activities, based on their perception on the e-Therapy sessions where eSchi was used. Therapists considered eSchi as a valuable tool to be used in their e-Therapy sessions and even requested that improvements were included in future versions. This includes new and more challenging activities. When both profiles (therapist and patient) are compared, it is obvious that the satisfaction level after using eSchi is superior for patients. This can be explained by the fact that therapists had higher expectations regarding the evolution of the tool, and the satisfaction score of using the system was strongly influenced by this fact.

The following topics depict the lessons learned in our study:

\subsection{Plan the User Centered Process}

The eSchi system is the resulting product of an e-Therapy project conceived by a multidisciplinary team with health professionals (HP Team) and information systems 
analysts, designers and developers (IT Team). The HP Team was composed by health professionals of the Hospital Sant Joan de Déu (HSJD), in Barcelona, and the IT Team by analysts, designer and developers of the Polytechnic University of Catalonia (UPC) Multimedia Engineering PhD Group of the Polytechnic Institute of Leiria. Both teams shared the dream to improve the quality of life of schizophrenic patients and the quality of their therapy sessions while recurring to new information and communication technologies.

The e-Therapy project started back in 2006, previously to the work here described. It evolved through a series of interviews and meetings to discuss the best way to approach the issues at hand.

\subsection{Specify the Context of Use and Specify User and Organizational requirements}

There were many informal conversations and meetings to conduct a contextual inquiry before the first focus group session actually occurred. In this first official meeting, the initial requirements for the system were defined. The meeting was recorded on camera and later a written transcript was made.

The focus group recognized the system's magnitude and complexity and settled in a prototype approach to the system that complied with the first basic functionalities that could be provided to both patients and the health professionals. Thus, an initial web portal with only two profiles would be available: patient and health professional. Health professionals would be able to manage their patients and their patients' therapy sessions. For each session, they could choose from the set of existing multimedia activities. As far as the patients were concerned, they could conduct a session with the activities that were defined by their health professionals. An aspect that was considered relevant was feedback information. Thus, every user should be able to visualize statistical information on the sessions' performance with distinct detail levels.

It was established that the HP Team would provide the contents for the specific therapy activities to be developed under the system and that the IT team would be responsible for the design and development of the system's architecture.

\subsection{Produce Design Solutions}

In this phase of development of the system, we designed it according to the knowledge we had gathered in previous phases. After an initial set of focus group meetings it was extremely important to include all the stakeholders including final users as a part of the entire process; to listen and take into consideration their ideas and professional remarks.

Hence, the system was defined in simple UML diagrams that included the results of the meetings. The diagrams were accepted and understood by all the participants in the focus group. This allowed the non-health professional members of the team to have a more accurate perception on the aspects of a classical therapy session, including its real surroundings. 
After this and still in this phase, the development team presented a prototype of the features implemented and collected feedback from the health team concerning the usability and features provided by the system. Several ad hoc tests were conducted to enable a participatory design.

In order to collect data from the study, both questionnaires and the eSchi system itself - as the monitoring tool - were used. The custom questionnaires used, besides obtaining an initial set of demographic data, were intended to obtain users' expectations before using an e-Therapy tool and their opinion/satisfaction after using the eSchi system. Two valid instruments were used during the studies: the After Scenario Questionnaire (ASQ) and the Computer System Usability Questionnaire (CSUQ). Usage data of the eSchi system was collected in an automatic and transparent way. All the questionnaires used, the custom questionnaires, the ASQ and the CSUQ were carefully translated into the users' native tongue, either to Spanish and Portuguese. Upon request by the health team and agreement inside the whole team, both the ASQ and CSUQ questionnaires were slightly adjusted to be applied to patients. The idea was to provide a clear questionnaire that could be easily understood and answered directly by patients. These adaptations were tested and validated by the health team. All the translations were verified and validated by the medical team inside the development team. Besides, the application was conceived to accommodate multilanguage support and became available in both languages.

To assure confidentiality of the study applicants, prevent possible personal detection upon data analysis and maintain the integrity of the information being gathered in the study, a unique codename was given to each user. The codenames endorsed were, for the simplicity of the process, also used as the usernames to login in the eSchi system. Hence, the real names of the users are not known and the only person that can establish the mapping is the psychiatrist, which is obliged to respect the standard patient-doctor confidentiality rules.

\subsection{Evaluate Design against User Requirements}

In order to obtain some adequate and formal feedback before deploying eSchi into a real setting, a Heuristic Evaluation (HE) was conducted.

Despite several usability techniques have been scientifically proven to be more adequate and better, as far as results are concerned, they are more time and money consuming than this "discount usability engineering" approach. It provides a more agile method to keep track of usability problems, directly applied to iterative development projects and based in early user-focus [36].

A test scenario was created to evaluate eSchi's patient module usability. Seven evaluators were asked to conduct a heuristic evaluation to the module and the information needed to conduct the evaluation was sent to each one. Each evaluator was asked to provide a written report with the issues examined, severity levels and further observations they considered useful. A new release of the system was published [41] and the system was considered ready to be deployed in a real setting.

The functional tests that mapped each user requirement to the actual features of the system were conducted directly in the field with the final users - patients and therapists. 
Some special considerations were taken regarding the user selection for the studies. In Barcelona, only one psychiatrist applied to take part in the study: the supervisor for the schizophrenia ward in the hospital. She made the patients' selection using as a major criterion the need for cognitive rehabilitation. Hence, the inclusion in the group of users was made upon consideration of having a positive impact on a patient's treatment, as the psychiatrist's personal communication referred. In Porto, only one therapist applied to take part in the study: the leading person of the occupational therapy section of the hospital. She made the patients' selection using as a major criterion the need for cognitive rehabilitation. Hence, the inclusion in the group of users was made upon consideration of being positive for a patient's treatment, as the therapist referred in the personal communication.

The first step to enable the study enrolment was the establishment of a physical scenario where the study could occur. In Barcelona, an ICT laboratory was established, where a limited number of computers, three, became preferred eSchi access points. Each desktop computer was equipped with a keyboard, a mouse and a set of headphone devices. The laboratory was located in the hospital's ICT room that has a free access policy for most patients.

In Porto, the hospital has an ICT room that has a limited access policy: only patients engaged in computer related activities can access the room and under a specific schedule. There is always the supervision of an informatics technician in the free access periods. It was guaranteed that each desktop computer could become an eSchi access point. This scenario setting was easier to conduct than the first one in Barcelona.

By the end of the first week of usage of the eSchi system in Barcelona, with the psychiatrist conducting sessions with the patients, several modifications to the application were requested. The suggestions came directly from the psychiatrist and revealed the patients' reactions while using the eSchi system. According to the psychiatrist, some users were highly sedated and were unable to click the mouse buttons in an autonomous way. It was suggested that the application received key strokes from the keyboard and allowed a better flow of the activities. The updates made to the application concerned the usage of the mouse and keyboard enabling a more efficient use of the system. The changes were implemented and readily applied, and, at the beginning of the second week, the users started using the new version.

By the end of the first week of a complete engagement from the users in the usage of eSchi in Porto, conducting sessions, the study went through a pause. According to the therapist, one of the users was discharged of the services, thus leaving the program and the other users required a higher level of complexity for the activities proposed. Hence, the technical team proposed a new set of activities to the therapist. This was the turning point in the deployment and evolution of the eSchi system, which allowed us to end our study. According to the therapist, it was time to develop and deploy new activities.

To conclude, we find that there are two important aspects that endorse the success of eSchi. Firstly, all the users that engaged in the study asked for further enhancements of the system, requesting new and improved activities. This reveals their interest in keeping on using the system. Secondly, eSchi is currently in use in a third site of the Sant Joan de Déu Institution by a third group of new users. This 
application of the system occurred through a professional recommendation of the therapist of the first site. The psychiatrist found eSchi suitable for her professional usage (according to the questionnaires' results) but also worth of recommendation for other professionals in the area.

The successful deployment of eSchi can be partly attributed to the Patient Centered Design (PCD) approach used. It started by listening to the patient's needs and requirements besides considering the social and technical context for the implementation of the product. The method implied the active engagement of endusers in all the design phases starting from early in the lifecycle development process. The PCD approach increased the communication between the health professionals that were involved in the project and the development team as the topics described above express.

\section{References}

1. Åborg, C., Sandblad, B., Gulliksen, J. et al.: Integrating Work Environment Considerations into Usability Evaluation methods-the ADA Approach. Interact Comput, 15 (2003) 453-471

2. Anderson, C.: Thumbnail: Susan Dray. 2010 (2007)

3. Asaro, P. M.: Transforming Society by Transforming Technology: The Science and Politics of Participatory Design. Accounting, Management and Information Technologies, 10 (2000) 257-290

4. Bevan, N., \& Curson, I.: Planning and Implementing User-Centred Design. (1999) 137-138

5. Beyer, H., \& Holtzblatt, K.: Contextual design: Defining customer-centered systems. Morgan Kaufmann Publishers Inc, San Francisco, CA, USA (1998)

6. Bias, R. G., \& Mayhew, D. J.: Cost-justifying usability. Academic Press, Inc, Orlando, FL, USA (1994)

7. Castelnuovo, G., Gaggioli, A., Mantovani, F. et al.: From Psychotherapy to e-Therapy: The Integration of Traditional Techniques and New Communication Tools in Clinical Settings. Cyberpsychology \& Behavior, 6 (2003a) 375-82

8. Catarci, T., Matarazzo, G., Raiss, G.: Driving Usability into the Public Administration: The Italian Experience. International Journal of Human-Computer Studies, 57 (2002) 121-138(18)

9. Chimero, F.: People Ignore Design that Ignores People. (2008)

10. Chinman, M., Hassell, J., Magnabosco, J. et al.: The Feasibility of Computerized Patient SelfAssessment at Mental Health Clinics. Administration and Policy in Mental Health and Mental Health Services Research, 34 (2007) 401-409

11. Clemensen, J., Larsen, S. B., Kyng, M. et al.: Participatory Design in Health Sciences: Using Cooperative Experimental Methods in Developing Health Services and Computer Technology. Qualitative Health Research, 17 (2007) 122-130

12. Cooper, A.: The inmates are running the asylum: Why high tech products drive us crazy and how to restore the sanity. 2nd edn. Pearson Higher Education (2004)

13. Dabbs, A. d. V., Myers, B. A., MC Curry, K. R. et al.: User-Centered Design and Interactive Health Technologies for Patients. Computers, Informatics, Nursing, 27 (2009) 175-183 10.1097/NCN.0b013e31819f7c7c

14. Demiris, G., Afrin, L. B., Speedie, S. et al.: Patient-Centered Applications: Use of Information Technology to Promote Disease Management and Wellness. A White Paper by the AMIA Knowledge in Motion Working Group. Journal of the American Medical Informatics Association, 15 (2008) 8-13

15. Earthy, J., Jones, B. S., Bevan, N.: The Improvement of Human-Centred Processes---Facing the Challenge and Reaping the Benefit of ISO 13407. Int. J. Hum. -Comput. Stud., 55 (2001) 553-585

16. Elvevag, B., Maylor, E. A., Gilbert, A. L.: Habitual Prospective Memory in Schizophrenia. BMC Psychiatry, 3 (2003) 9

17. Freire, C. S., Reis, C. I., Monguet, J. M.: ESchi - A Multimedia Portal for Schizophrenia Learning and Rehabilitation: Work in Progress. (2008) 2175-2180

18. Google User Experience Team: Google User Experience. 2010 (2010) 
19. Greenbaum, J.: A Design of One’s own: Towards Participatory Design in the United States. In: Schuler, D. and Namioka, A. (eds.) Participatory Design Principles and Practices., pp. 27-27-37. Lawrence Erlbaum Associated, Inc, New Jersey (1993)

20. Hix, D., \& Hartson, H. R.: Developing user interfaces: Ensuring usability through product \& process. John Wiley \& Sons, Inc, New York, NY, USA (1993)

21. ISO: ISO 9241-1:1997 - Ergonomic Requirements for Office Work with Visual Display Terminals (VDTs) -- Part 1: General Introduction. 2010 (2009)

22. ISO: ISO 9241-11:1998 - Ergonomic Requirements for Office Work with Visual Display Terminals (VDTs) -- Part 11: Guidance on Usability. 2010 (1998)

23. Johansson, M. C. G.: Social and Organizational Aspects of Requirements Engineering Methods. A Practice-Oriented Approach. (1999)

24. Jokela, T.: Making User-Centred Design Common Sense: Striving for an Unambiguous and Communicative UCD Process Model. (2002) 19-26

25. Jokela, T., Iivari, N., Matero, J. et al.: The Standard of User-Centered Design and the Standard Definition of Usability: Analyzing ISO 13407 Against ISO 9241-11. (2003) 53-60

26. Jones, R. B., Atkinson, J. M., Coia, D. A. et al.: Randomised Trial of Personalised Computer Based Information for Patients with Schizophrenia. BMJ, 322 (2001) 835-840

27. Karat, C.: Cost-justifying usability engineering in the software life cycle. In: Helander, M., Landauer, T.K. and Prabhu, P. (eds.) Handbook of Human Computer Interaction. Elsevier, Amsterdam (1997)

28. Kitchenham, B., Pickard, L., Pfleeger, S. L.: Case Studies for Method and Tool Evaluation. IEEE SOFTWARE, 12 (1995) 52-62

29. Kushniruk, A. W., \& Patel, V. L.: Cognitive and Usability Engineering Methods for the Evaluation of Clinical Information Systems. J. Biomed. Inform., 37 (2004) 56-76

30. Liberman, R. P., Hilty, D. M., Drake, R. E. et al.: Requirements for Multidisciplinary Teamwork in Psychiatric Rehabilitation. Psychiatric Services, 52 (2001) 1331-1342

31. Maguire, M.: Methods to Support Human-Centred Design. Int. J. Hum. -Comput. Stud., 55 (2001) 587634

32. Mayhew, D. J.: The Usability Engineering Lifecycle. (1999) 147-148

33. MedlinePlus: Medical Encyclopedia - Schizophrenia. 2008 (2008) 1

34. Newman, M. G., Consoli, A., Taylor, C. B.: Computers in Assessment and Cognitive Behavioral Treatment of Clinical Disorders : Anxiety as a Case in Point : Cost-Effectiveness, Cost-Benefits, and Quality of Life. Behavior Therapy, 28 (1997) 211-235

35. Nielsen, J.: Usability engineering. 1st edn. Morgan Kaufmann, San Francisco, USA (1993)

36. Nielsen, J.: Guerrilla HCI: Using Discount Usability Engineering to Penetrate the Intimidation Barrier. Guerrilla $\{\mathrm{HCI}\}$, (1994) 245-272

37. NykÄNEN, P.: Requirements for User-Friendly Personal Health Information Systems. In: Bos, L., Blobel, B., Marsh, A., et al (eds.) Studies in Health Technology and Informatics, vol. 137 (2008)

38. Pare, G., Sicotte, C., Jaana, M. et al.: Prioritizing the Risk Factors Influencing the Success of Clinical Information System Projects. A Delphi Study in Canada. Methods of Information in Medicine, 47(3) (2008) 251-251-9.

39. Postel, M. G., de Haan, H. A., De Jong, C. A. J.: E-Therapy for Mental Health Problems: A Systematic Review. Telemedicine and e-Health, 14 (2008) 707-714

40. Rahimi, B., \& Vimarlund, V.: Methods to Evaluate Health Information Systems in Healthcare Settings: A Literature Review. Journal of Medical Systems, 31 (2007) 397-432

41. Reis, C. I., Freire, C. S., Fernandez, J. et al.: ESchi - an e-Therapy Tool for Rehabilitation and Training. (2009)

42. Rosson, M. B., \& Carroll, J. M.: Usability engineering: Scenario-based development of humancomputer interaction. Morgan Kaufmann Publishers Inc, San Francisco, CA, USA (2002)

43. Rothschild, A. S., Dietrich, L., Ball, M. J. et al.: Leveraging Systems Thinking to Design PatientCentered Clinical Documentation Systems. Int. J. Med. Inf., 74 (2005) 395-398

44. Samaras, G. M., \& Horst, R. L.: A Systems Engineering Perspective on the Human-Centered Design of Health Information Systems. J. Biomed. Inform., 38 (2005) 61-74

45. Schmidt, R., Lyytinen, K., Keil, M. et al.: Identifying Software Project Risks: An International Delphi Study. Journal of Management Information Systems, 17 (2001) 5-36

46. Sjöberg, C., \& Timpka, T.: Participatory Design of Information Systems in Health Care. Journal of the American Medical Informatics Association, 5 (1998) 177-183

47. Smuc, M., Mayr, E., Lammarsch, T. et al.: To Score Or Not to Score? Tripling Insights for Participatory Design. IEEE Comput.Graph.Appl., 29 (2009) 29-38 
48. Spokoiny, A., \& Shahar, Y.: A Knowledge-Based Time-Oriented Active Database Approach for Intelligent Abstraction, Querying and Continuous Monitoring of Clinical Data. Studies in Health Technology and Informatics, 107 (2004) 84-8.

49. UPA: Designing the User Experience. (2010)

50. Walker, H.: Computer-Based Education for Patients with Psychosis. Nursing Standard, 20(30) (2006) 49-49-56.

51. Wijk, J. J. v.: Views on Visualization. IEEE Trans. Visual. Comput. Graphics, 12 (2006) 421-433

52. World Health Organization: Improving Health Systems and Services for Mental Health. 2010 (2009) 104

53. World Health Organization: MhGAP - Mental Health Gap Action Programme. Scaling Up Care for Mental, Neurological, and Substance use Disorders. 2010 (2008)

54. World Health Organization: Facts. 2010 (2007)

55. World Health Organization: The Global Burden of Disease: 2004 Update. 2010 (2004)

56. Yin, R. K.: Case Study Research: Design and Methods. In: Anonymous Applied Social Research Methods, vol. 5, pp. 181. Sage Publications, Thousand Oaks, California, USA (2002)

57. Yin, R. K.: Applications of case study research. Applied Social Research Methods, Vol. 34. 2nd edn. Sage Publications, Thousand Oaks, California, USA (2003) 